\title{
Electronic Specific Heat of Iron-based Dilute Alloys
}

\author{
By Samuel S. Shinozaki*
}

\begin{abstract}
The change in the electronic specific heat of dilute alloys, especially iron with a small addition of solute elements, was investigated experimentally in order to understand the electronic band structure of transition elements in relation to the origin of ferromagnetism. Since we have to measure a small change (order of one or two percent) in the electronic specific heat with a small addition of the solute element, a special calorimeter, which is used to measure simultaneously the specific heat of three samples, one pure metal and two alloys, has been developed for this purpose. Using this calorimetry, the change of $\gamma$ can be measured with higher accuracy in one order of magnitude. The solute elements for iron-based alloys are: $\mathrm{Ti}, \mathrm{V}, \mathrm{Cr}, \mathrm{Mn}, \mathrm{Co}, \mathrm{Ni}, \mathrm{Nb}, \mathrm{Mo}, \mathrm{W}, \mathrm{Si}$ and $\mathrm{Al}$. Silver-based alloys with gold are also measured to check the accuracy of this calorimeter. The variation of $\gamma$ for $\mathrm{Ag}(\mathrm{Au})$ alloys is less than experimental uncertainty, while the variation of the Debye temperature shows a linear decrease with gold concentration. For iron-based alloys, out of the eleven solutes, six decrease the $\gamma$ values linearly with solute concentration, $c$. Their values of $(1 / \gamma) \Delta \gamma / \Delta c$ are: $\mathrm{Fe}(\mathrm{Ti}),-1.0 ; \mathrm{Fe}(\mathrm{V}),-2.2$; $\mathrm{Fe}(\mathrm{Cr}),-2.0 ; \mathrm{Fe}(\mathrm{Co}),-0.6 ; \mathrm{Fe}(\mathrm{Mo}),-0.8 ;$ and $\mathrm{Fe}(\mathrm{W}),-2.4$. Four solutes increase the $\gamma$-values nonlinearly with respect to the solute concentration. The average values of $(1 / r) \Delta r / \Delta c$ near $c=0$ are: $\mathrm{Fe}(\mathrm{Al}),+1.2 ; \mathrm{Fe}(\mathrm{Si}),+0.6 ; \mathrm{Fe}(\mathrm{Mn}),+2.0$ and $\mathrm{Fe}(\mathrm{Ni}),+2.6$. These data are utilized to discuss the applicability of the rigid band model as well as to correlate the changes with those of magnetic properties.
\end{abstract}

(Received August 29, 1967)

\section{Introduction}

Recently, there have been attempts to describe the electronic states of an impurity atom in a host metal using the results of specific heat measurements at low temperature. In particular, the question of the applicability of a rigid band model to dilute alloys has been investigated by several workers ${ }^{(1) \sim(5)}$. From the

* Scientific Laboratory, Ford Motor Company, Dearborn, Michigan, U.S.A..

(1) J. A. Rayne: Phys. Rev., 108 (1957), 22.

(2) G. L. Guthrie, S. A. Friedberg and J.E.Goldman : Phys. Rev., 113 (1959), 45. results of many electronic specific heat measurements on the transition metals and their isostructural solid solutions, Cheng, Wei and Beck ${ }^{(6)}$ showed that the results can be interpreted, to a rough approximation,

(3) G. L. Guthrie: Phys. Rev., 113 (1959), 793.

(4) H. J. Blythe, T. M. Holden, M. Dixon and F. E. Hoare: Phil. Mag., 11 (1965), 235.

(5) D. A. Dicke and B. A. Green, Jr.: Phys. Rev., 153 (1967), 800.

(6) C. H. Cheng, C. T. Wei and P. A. Beck: Phys. Rev., 120 (1960), 426. See also later works. E. A.Stark, Jr., C. H. Cheng and P. A. Beck: Phys. Rev., 126 (1962), 1746. K. P. Gupta, C. T. Wei and P. A. Beck: J. Phys. Chem. Solids, 25 (1964), 759; N. Pessall and K. P. Gupta: J. Phys. Chem. Solids, 25 (1964), 993. 
within the framework of the rigid band model. Their results, however, show that there is an upward cusp in electronic specific heat coefficient, $\gamma$, versus $\vec{Z}$ (average number of electrons) curve at pure $\mathrm{Fe}$. The cusp at the pure metal cannot be explained by the rigid band model. This phenomenon peculiar to pure metal motivated us to investigate the $\mathrm{Fe}$-based dilute alloys. Furthermore, $\mathrm{Fe}$ has several advantages as a host metal for the study of dilute solution alloys, as reviewed by Arrott and Noakes ${ }^{(7)}$. With $\mathrm{Fe}$, it is relatively easy to prepare many different alloys and to carry out chemical analyses. $\mathrm{Fe}$ also has a large value of $\gamma$ in comparison with noble metals. All of the samples used in this experiment are taken from the same ingots as used by Arrott and Noakes ${ }^{(7)(8)}$ for measurements of lattice parameters, spontaneous magnetization Curie temperature, high temperature susceptibility and resistivity. This allows a direct comparison of magnetic and other properties with the specific heat data which may give an important insight into the origin of ferromagnetism of iron.

Here, we have measured the specific heat of these alloys at low temperature. The typical concentrations of the solutes are one and two atomic percent. Even though the concentrations are not truly dilute, they are as dilute as the accuracy of our measurements would justify. The solutes used in this experiment are other elements of the first transition series, Mo and $W$, which represent second and third transition elements and also a variation on a downward column in the periodic table from $\mathrm{Cr}$, and finally $\mathrm{Si}$ and $\mathrm{Al}$ as examples of nontransition metals.

The small changes in the specific heat of these dilute alloys are almost the same order of magnitude as experimental uncertainty. Thus, in order to obtain some reasonable relationship between the change in electronic specific heat coefficient $\gamma$ and the solute concentration $c$, a new method of simultaneous calorimetry was developed to accomplish this purpose. The simultaneous calorimeter contains three samples, one pure metal and two dilute alloys. Although the individual measurement of the specific heat is a conventional technique, a gain in relative accuracy is achieved by the simultaneous measurement of the three samples. It should also be pointed out that the simultaneous measurement can be carried out as easily as the single sample measurement.

The final results of our measurements are analyzed in connection with the recent theoy of the density of states of the dilute alloys by Brailsford ${ }^{(9)}$. The results are also compared with other measurements by Arrott and Noakes to find some correlations with other properties.

\section{Apparatus and Procedure}

The basic principle in the design of the calorimeter

(7) A. Arrott and J. E. Noakes in Iron and Its Dilute Solid Solutions (Interscience Publishers, Inc., New York (1963), p.p. $81 \sim 117$.

(8) J. E. Noakes and A. Arrott : J. Appl. Phys., 37 (1966), 1264.

(9) A. D. Brailsford: Proc. Roy. Soc. (London), 292 A (1966), 433. is the complete symmetry of the whole system, which includes three samples, sample holders, carbon thermometers, heater coils, superconducting lead wires, cotton thread supports and thermal switches. A rough sketch of the calorimeter is shown in Fig. 1. Each part

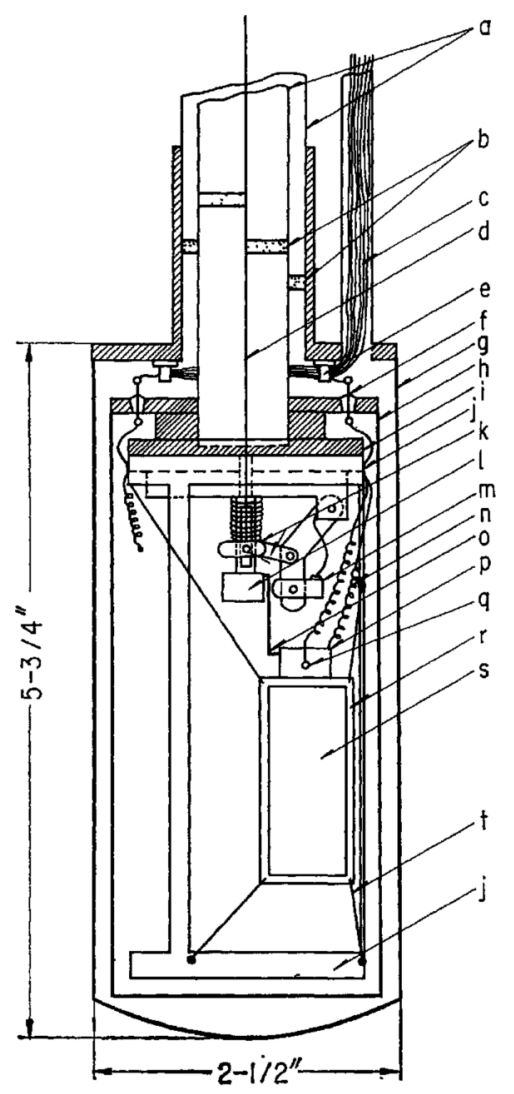

Fig. 1 The two concentric calorimeter can (g and h) are shown in cutaway view while the support frame (j), part of the heat switch (d, $\mathrm{k}, \mathrm{l}, \mathrm{m}$ and $\mathrm{o}$ ) with one of the movable jaws (m) and one sample holder (r) are shown in direct view.

of the calorimeter is described in detail elsewhere ${ }^{(10)}$, and some of the methods of data analysis adopted here and a part of the results have already been published (11). The sample holders ( $r$ ) are supported very rigidly with seven pieces of cotton thread $(t)$ in such a way that the holders should have the same response to outside vibrations. All of the superconducting lead wires (n) and cotton threads do not touch one another and they are not disturbed at all when a sample is placed into a space (s). In order to avoid the problem caused by the helium exchange gas, thermal switches ( $\mathrm{d}, \mathrm{k}, \mathrm{l}, \mathrm{m}$ and $\mathrm{o}$ ) are constructed. The symmetry of the thermal switches is achieved by three movable copper jaws (m), which press the copper wire (o) of the sample holder to the central stationary post (l). With the symmetric thermal switches, the initial heating rate upon opening the thermal switch are the same. Thus, the temperatures of the three samples stay very close to each other at the beginning of the measurement.

Another major source of error comes from the

(10) S. Shinozaki : Technical Report of Scientific Laboratory, Ford Motor Company. Report No. SL 67-71.

(11) S. Shinozaki and A. Arrott : Phys. Rev., 152 (1966), 611. 
uncertainty in the calibration of the secondary thermometer (q) (1/10 watt, 47-ohms, Allen-Bradley carbon resistor) against the vapor pressure of liquid helium. The calibration is affected by the range of temperature, number of calibration points and zero point reading of the mercury manometer. Using the technique suggested by Hoare and Yates ${ }^{(12)}$, helium gas is liquified in the inner calorimetric can (h) which surrounds the sample holders with their carbon thermometer (q) built into it. Thus, the calorimeter can itself is used as a helium vapor pressure bulb, which is isolated from the helium bath by an outer can (g). The liquified helium is pumped on very slowly. Thus, the sample holders are placed at the vapor-liquid interface, which is the coldest place in the calorimeter. The measured vapor pressure corresponds to that of the interface. There is an instantaneous response of the thermometer to the changes in vapor pressure. The three thermometers are thus calibrated at the same temperature and the systematic errors in the thermometry are cancelled out.

The main features of the calorimetry are thus: a symmetric arrangement of the three sample holders with attached heaters and thermometers, a three-jawed mechanical thermal switch, use of the inner calorimetric can as a vapor pressure bulb, the simultaneous measurements of the heat capacity for the three samples and the simultaneous calibration of the three carbon resistor thermometers.

The resistance measurement for the carbon thermometer is made by the standard technique of four terminals with a Tinseley Vernier potentiometer and a Leeds and Northrup d.c. microvoltmeter. In the specific heat measurement, the potentiometer is used for voltage suppression. The imbalance is amplified by the d.c. microvoltmeter and is recorded on a high pen speed, $10 \mathrm{mV}$, Western chart recorder. The sensitivity of this system is \pm 0.1 microvolt. The current used in this thermometer system is supplied from a constant voltage source rather than a constant current source. The current is first adjusted to be 3.0 microamperes when the temperature of the sample is at $4.2^{\circ} \mathrm{K}$. The stability of the constant voltage source is checked by substituting a dummy precision resistor for the carbon resistance thermometer. The current used in the heating circuit is monitored continuously as it flows either into the heater coil on the holder or into a dummy resistor whose resistance is equal to the total resistance of the heater circuit at $4.2^{\circ} \mathrm{K}$.

During the calibration of the carbon thermometer, helium gas is liquified in the inner calorimeter can and the vapor pressure of the liquid helium is measured with an automatic manometer. In the first series of the experiments, an Exactel Servomanometer was used, but later, both the Exactel Servomanometer and a fused quartz pressure gage made by Texas Instrument were used. The Exactel Servomanometer is a well and cistern type manometer with a float in a precision bore quartz tubing. The position of the float is sensed by

(12) F. E. Hoare and B. Yates: Proc. Roy. Soc. (London), 240 A (1957), 421. a differential transformer which is operated by a servo system. In this system, the accuracy of the pressure reading is thus dependent on the position of the float which is greatly affected by the impurity on the surface of the mercury, and also is dependent on the accurate reading of the zero point. On the other hand, by using the fused quartz pressure gage most of the difficulties of the mercury manometer were eliminated.

The calibration of the thermometers is thus carried out rapidly. For the pressure is stabilized and the temperature of the samples reaches the reference temperature quickly. This calibration procedure is proved to be adequate by the observation that there is no discontinuity in the calibration curve at the $\lambda$ point of liquid helium (where there are some drastic changes in the properties of liquid helium).

\section{Analysis of Data and Results}

Each thermometer is calibrated at the same 22 temperatures between $2^{\circ} \mathrm{K}$ and $4.2^{\circ} \mathrm{K}$. Calculation of the resistance-temperature relations is programmed for a Philco 2000 computer using the 1958 international table for the vapor pressure of liquid helium. The data are fitted to an empirical equation

$$
\left[\log \left(R / R_{0}\right) / T\right]^{1 / 2}=A+B \log \left(R / R_{0}\right)+\mathrm{C}\left[\log \left(R / R_{0}\right)\right]^{2},
$$

where the three parameters $A, B$ and $C$ are adjusted in such a way as to minimize the quantity $\Sigma\left(T_{\exp }-T_{\text {cal }}\right)_{i}^{2}$, with $R$ in ohms and $R_{0}=8 \mathrm{ohms}$. All of the points are fitted consistently within 0.5 millidegree, using the fused quartz manometer. As the thermometers are calibrated at the same set of temperatures, the systematic uncertainties in the thermometers are all cancelled out.

The changes in temperature of the samples, $\Delta T$, which is caused by the measured electrical energy input, $I^{2} R T$, is determined by the standard technique of extrapolation of a resistance tersus time curve from the results before and after a heating period. The three records of the temperature drift versus time are almost parallel to one another (the temperature of the three samples stays very close to each other from the beginning to the end of an entire run). This makes it very easy to detect any disturbance during the specific heat measurement.

Although it is not the primary purpose of these experiments to measure the specific heat of pure metals, we compare our results for Ag of high purity (99.9999 $\%$ ) and $\mathrm{Fe}$ with the recent results of other workers to confirm the reliability of our measurement. Our result of $\mathrm{Ag}\left(\gamma=0.656 \mathrm{mj}-\mathrm{deg}^{-1}-\mathrm{mole}^{-2}, \theta_{D}=225.3^{\circ} \mathrm{K}\right)$ shows a very good agreement with the most recent measurement of a high purity $\mathrm{Ag}$ by Green and Valladares ${ }^{(13)}$, which shows $\gamma=0.654 \mathrm{mj}-\mathrm{deg}^{-2}-\mathrm{mole}^{-1}$ and $\theta_{D}=225.8$ ${ }^{\circ} \mathrm{K}$. The results of all measurements on the pure $\mathrm{Fe}$ are shown in Table 1 . The results in groups $I$ and II have a larger fluctuation in $\gamma$ value than the ones in group III. This fluctuation and increase in $\gamma$ value are due to the improvements of our measuring system,

(13) B. A. Green, Jr. and A. A.Valladeres: Phys. Rev., 142 (1966), 379 . 
especially the reduction/of uncertainty in the thermometer calibration with the introduction of the quartz manometer. Thus, the values of group III should be more accurate. The average value of $\gamma$ in group III is about $1 \%$ higher than the value obtained by Dixon alloy $x$ where a is a specification of this method, are listed in Table 1.

The second method is intended to make a point by point comparison between the data of the pure metal and those of the alloys, taking advantage of the

Table 1 Results for iron and iron alloys.

\begin{tabular}{|c|c|c|c|c|c|c|c|c|c|c|c|c|}
\hline \multirow{2}{*}{\multicolumn{2}{|c|}{ Experimental }} & \multirow{2}{*}{\multicolumn{4}{|c|}{$C=\gamma T+\beta T^{3}\left[\mathrm{mj} /{ }^{\circ} \mathrm{K}-\mathrm{mole}\right]$}} & \multicolumn{7}{|c|}{$\theta_{D}=\left(\frac{1943}{\beta}\right)^{1 / 3}\left[{ }^{\circ} \mathrm{K}\right]$} \\
\hline & & & & & & $1 \mathrm{~s}$ & alloys & & & 2nd all & & \\
\hline Name & Group & $\gamma$ & $\beta$ & $\theta D$ & $\begin{array}{l}\text { Atomic } \\
\text { Percent }\end{array}$ & $\Delta \gamma_{1}(\mathrm{a})$ & $\Delta \beta_{1}(\mathrm{a})$ & $\Delta r_{1}(\mathrm{~b})$ & $\begin{array}{l}\text { Atomic } \\
\text { percent }\end{array}$ & $\Delta r_{2}(\mathrm{a})$ & $\Delta r_{2}(\mathrm{a})$ & $\Delta \gamma_{2}(\mathrm{~b})$ \\
\hline $\left.\begin{array}{c}\text { 1st } \mathrm{Si} \\
\text { 2nd } \mathrm{Si} \\
\mathrm{Al} \\
\text { 1st } \mathrm{Ni} \\
\text { 2nd } \mathrm{Ni} \\
\mathrm{Co} \\
\text { 1st } \mathrm{Nb} \\
\text { 2nd } \mathrm{Nb} \\
\text { 1st W } \\
\text { 2nd } \mathrm{W} \\
\text { 3rd } \mathrm{Mo} \\
\text { 1st } \mathrm{Mo} \\
\text { 2nd } \mathrm{Mo} \\
\text { 1st } \mathrm{Cr} \\
\text { 2nd } \mathrm{Cr} \\
\text { 1st } \mathrm{V} \\
\text { 2nd } \mathrm{V} \\
\text { 1st } \mathrm{Mn} \\
\text { 2nd } \mathrm{Mn} \\
\text { 1st } \mathrm{Ti} \\
\text { 2nd } \mathrm{Ti} \\
\text { 3rd } \mathrm{Ti}\end{array}\right\}$ & II & $\begin{array}{l}4.818 \\
4.812 \\
4.815 \\
4.800 \\
4.824 \\
4.818 \\
4.782 \\
4.788 \\
4.830 \\
4.826 \\
4.797 \\
4.809 \\
4.815 \\
4.776 \\
4.797 \\
4.767 \\
4.728 \\
4.772 \\
4.771 \\
4.799 \\
4.776 \\
4.807\end{array}$ & $\begin{array}{l}0.0191 \\
0.0197 \\
0.0197 \\
0.0209 \\
0.0189 \\
0.0184 \\
0.0194 \\
0.0210 \\
0.0181 \\
0.0189 \\
0.0198 \\
0.0185 \\
0.0172 \\
0.0217 \\
0.0213 \\
0.0266 \\
0.0254 \\
0.0231 \\
0.0227 \\
0.0212 \\
0.0243 \\
0.0201\end{array}$ & $\begin{array}{l}467 \\
462 \\
462 \\
453 \\
468 \\
472 \\
465 \\
453 \\
475 \\
468 \\
461 \\
472 \\
483 \\
448 \\
450 \\
418 \\
425 \\
438 \\
441 \\
451 \\
431 \\
459\end{array}$ & $\begin{array}{l}1.08 \\
1.04 \\
1.00 \\
0.89 \\
0.50 \\
0.31 \\
1.16 \\
0.46 \\
1.10 \\
1.14 \\
1.16\end{array}$ & $\begin{array}{r}0.029 \\
0.025 \\
0.066 \\
0.098 \\
0.103 \\
-0.017 \\
0.122 \\
0.139 \\
-0.046 \\
-0.044 \\
-0.035 \\
-0.038 \\
-0.050 \\
-0.53-0.051 \\
-0.034 \\
-0.106 \\
-0.114 \\
0.114 \\
0.119 \\
-0.067 \\
-0.042 \\
-0.069\end{array}$ & $\begin{array}{r}-0.0009 \\
-0.0010 \\
-0.0009 \\
0.0007 \\
0.0000 \\
-0.0007 \\
0.0016 \\
0.0025 \\
0.0006 \\
0.0010 \\
0.0002 \\
0.0004 \\
0.0019 \\
-0.0004 \\
-0.0024 \\
-0.0016 \\
-0.0002 \\
-0.0024 \\
-0.0021 \\
0.0008 \\
-0.0026 \\
0.0005\end{array}$ & $\begin{array}{r}0.025 \\
0.014 \\
0.058 \\
0.104 \\
0.104 \\
-0.025 \\
0.123 \\
0.138 \\
-0.044 \\
-0.041 \\
-0.043 \\
-0.035 \\
-0.036 \\
-0.055 \\
-0.049 \\
-0.117 \\
-0.112 \\
0.099 \\
0.103 \\
-0.059 \\
-0.061 \\
-0.062\end{array}$ & $\begin{array}{l}0.88 \sim 1.00 \\
2.19 \sim 2.29 \\
2.21 \sim 2.25 \\
2.32 \sim 2.36\end{array}$ & $\begin{array}{r}0.111 \\
0.088 \\
0.134 \\
0.483 \\
0.497 \\
-0.076 \\
0.271 \\
0.290 \\
-0.077 \\
-0.076 \\
-0.088 \\
-0.079 \\
-0.104 \\
-0.102 \\
-0.096 \\
-0.217 \\
-0.216 \\
0.231 \\
0.245 \\
-0.114 \\
-0.096 \\
-0.115\end{array}$ & $\begin{array}{r}-0.0023 \\
-0.0017 \\
0.0003 \\
0.0022 \\
-0.0002 \\
-0.0025 \\
0.0033 \\
-0.0000 \\
0.0005 \\
-0.0001 \\
0.0010 \\
0.0008 \\
0.0026 \\
0.0002 \\
0.0002 \\
-0.0023 \\
-0.0019 \\
-0.0015 \\
-0.0030 \\
0.0008 \\
-0.0016 \\
0.0004\end{array}$ & $\begin{array}{r}0.092 \\
0.082 \\
0.141 \\
0.506 \\
0.500 \\
-0.104 \\
0.294 \\
0.301 \\
-0.072 \\
-0.071 \\
-0.077 \\
-0.070 \\
-0.079 \\
-0.096 \\
-0.091 \\
-0.236 \\
-0.226 \\
0.225 \\
0.225 \\
-0.105 \\
-0.103 \\
-0.108\end{array}$ \\
\hline
\end{tabular}

et al. ${ }^{(14)}$, which is $\gamma=4.78 \mathrm{mj}-\mathrm{mole}^{-1}-\operatorname{deg} K^{-2}$ and $\theta_{D}$ $=464^{\circ} \mathrm{K}$.

The main aim in this experiment is to measure the change in the value of $\gamma$ due to alloying. We have tried two ways to analyze the data. The first method is to fit all of the data points between $2^{\circ} \mathrm{K}$ and $4^{\circ} \mathrm{K}$ to the equation $C=\gamma T+\beta T^{3}$ by using the ordinary least square fit method. In this case, all the data for the three samples are taken as if the sample were measured separately. An example of the results of the fit is shown in Fig. 2 by three straight lines for the

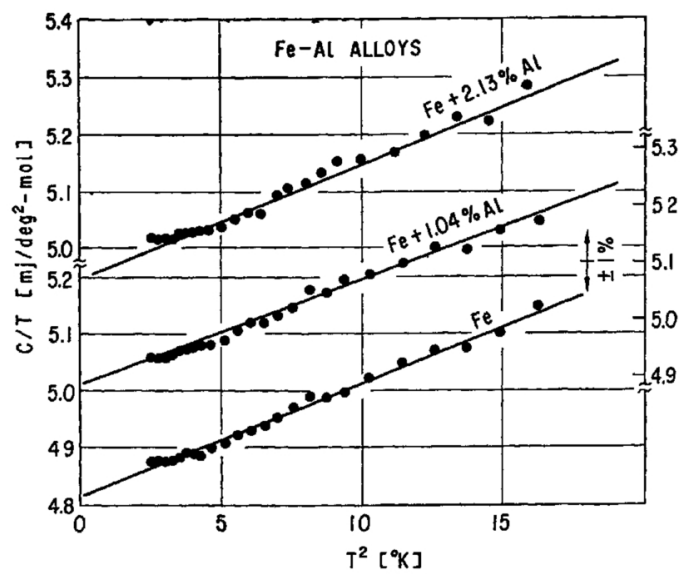

Fig. 2 Specific heat of $\mathrm{Fe}(\mathrm{Al})$ alloys.

plot $C / T$ versus $T^{2}$ for the three samples. The differences $\Delta \gamma_{x}(\mathrm{a})=\gamma_{x}-\gamma_{\mathrm{Fe}}$ and $\Delta \beta_{x}(\mathrm{a})=\beta_{x}-\beta_{\mathrm{Fe}}$ for an

(14) M. Dixon, F. E. Hoare, T. M. Holden and D. E. Moody : Proc. Roy. Soc. (London), 285 A (1965), 561. simultaneous measurement of the specific heat of the three samples. The temperatures of the alloys are slightly adjusted to the temperature of the pure metal for comparison. This process was done using a running average of five points. The differences are fitted to the equation $\Delta(C / T)=\Delta \gamma+\Delta \beta T^{2}$. Such results are shown in Fig. 3. The details of the fit of all the data are described in the technical report of the Ford Scientific Laboratory ${ }^{(10)}$. In this figure, the base line indicates the running average values of five points for the pure $\mathrm{Fe}$ sample. indicates the difference of

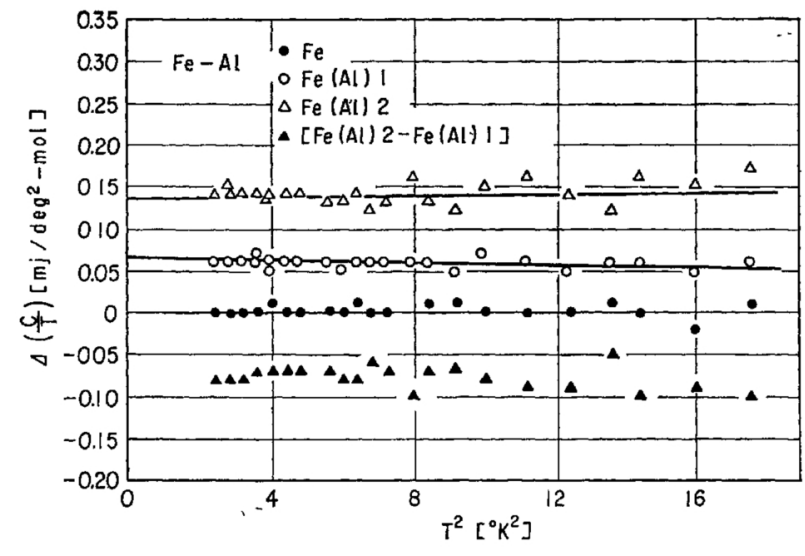

Fig. 3 Specific heat differences of $\mathrm{Fe}(\mathrm{Al})$ alloys from the pure $\mathrm{Fe}$ solvent. See text for detailed explanation.

the individual points of $\mathrm{Fe}$ from the base line. 0 and $\Delta$ indicate the direct differences in alloy (1) and $\mathrm{Fe}$ and alloy (2) and $\mathrm{Fe}$, respectively. Finally, $\Delta$ indicates the difference between alloy (1) and alloy (2). The straight 
lines are the results from the first method for comparison. Thus, the two different methods are in good agreement. Since the change in $\beta, \Delta \beta$, is small and rather difficult to determine, we have taken, as the third method specified by $b$, the average values of $\Delta \gamma_{1}$ (b) for alloy (1) and $\Delta \gamma_{2}$ (b) for alloy (2). This means that the change in $\beta$ is neglected. If we compare the several runs for the same sample, we find that the variation in the $\Delta \gamma(\mathbf{b})^{\prime}$ s is less than that of the $\Delta \gamma(\mathbf{a})^{\prime} \mathrm{s}$. This is because there is an additional degree of freedom, $\beta$, in the fit to obtain the $\Delta \gamma(\mathrm{a})^{\prime}$ s. Attempts to determine $\Delta \beta$ from elastic measurements were not successful.

Fig. 4 and Table 1 summarize our experimental

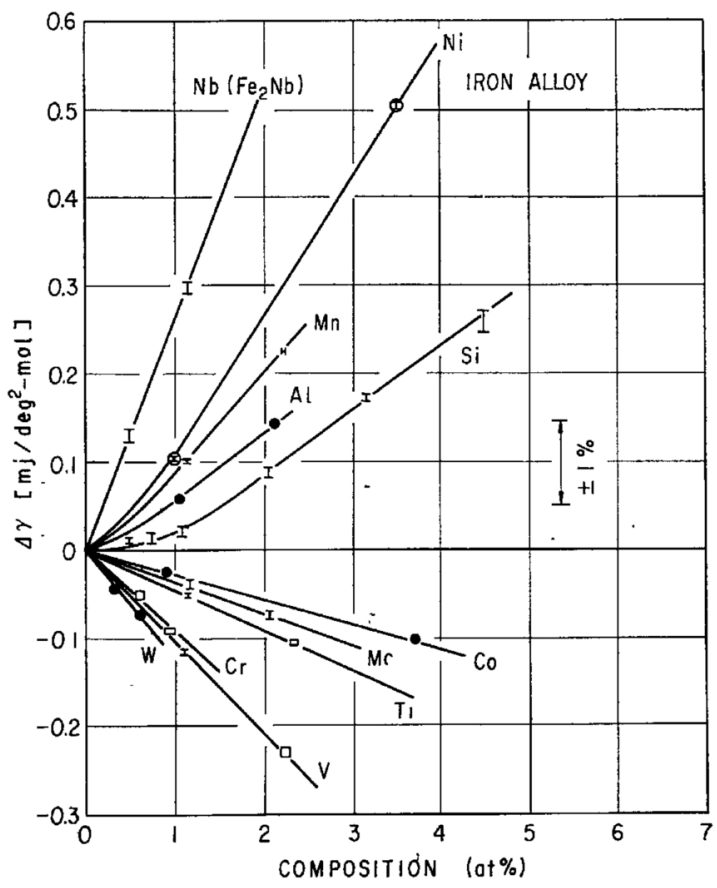

Fig. 4 Variation of $\gamma$ with concentration of solutes for dilute alloys of $\mathrm{Fe}$.

results of Fe-based alloys. Fig. 4 shows the change of $\gamma$ as a function of atomic concentration of the eleven solutes. The six solutes, Ti, V, Cr, Mo, W and Co, lower the $\gamma$ value of $\mathrm{Fe}$ in a linear relation with their atomic concentration. This group appears to be divided into two groups, that is, V, Cr and W for one group and $\mathrm{Ti}, \mathrm{Co}$ and Mo for another group. The average rate of change of $\gamma$ with solute concentration for the first group is about twice as large as the second group. The four solutes, $\mathrm{Mn}, \mathrm{Ni}, \mathrm{Al}$ and $\mathrm{Si}$, raise the $\gamma$ values of $\mathrm{Fe}$ in a nonlinear relation with solute concentration. The apparent nonlinearity does not seem to be the result of experimental uncertainty. The data for $\mathrm{Fe}(\mathrm{Nb})$ alloys are for two-phase alloys with a solubility limit of $0.1 \% \mathrm{Nb}$ in $\mathrm{Fe}$. This result cannot be compared with rest of the samples.

\section{Discussion}

The coefficient of the electronic specific heat, $\gamma$, is proportional to the density of states $N(\varepsilon)$ at the Fermi energy $\varepsilon_{F}$. When some solute is added to a pure solvent metal, we should be able to obtain the density of states at the Fermi energy for this alloy from the change in $\gamma$. In the rigid band model, these two densities of states are related with the same proportionality constant. However, experimental results indicate that such a simple model is not applicable when the concentration of solute is very dilute, as pointed out in the introduction. Recently, Brailsford has derived expressions for the change in the density of states in dilute alloys by keeping track of the change in energy of each of the many one electron states of the solvent during the addition of solute. The concentration of the solute is assumed to be dilute as long as the change in electronic specific heat is linear with the concentration of the solute. Brailsford's argument is repeated here for the sake of discussion.

When the solute is added to the solvent (pure metal), there is a shift in the energy of $k$ th state of the solvent. This, we denote by $\Delta\left(\varepsilon_{k}, k\right)$ where $\varepsilon_{k}$ is the energy in the $k$ th state. Let us assume then that the state in the alloy, corresponding to the state $\Psi_{k}$ of the periodic field of the solvent, has the energy $\left\{\varepsilon_{k}+\Delta\left(\varepsilon_{k}, k\right)\right\}$. Now, let $Z_{0}(\varepsilon)$ be the number of states below the energy $\varepsilon$ in the pure solvent. Then, as a result of alloying each state is shifted in energy by an amount $\Delta(\varepsilon)$. The number of states below the energy $\varepsilon$ in the alloy, $Z_{A}(\varepsilon)$, is given by

$$
Z_{A}(\varepsilon) \simeq Z_{0}(\varepsilon-\Delta(\varepsilon)) \simeq Z_{0}(\varepsilon)-N_{0}(\varepsilon) \Delta(\varepsilon),
$$

where the density of states $N_{0}(\varepsilon)$ is given by $N_{0}(\varepsilon)=$ $d Z_{0}(\varepsilon) / d \varepsilon$. If the level shift depends on $k$, the decrease in $Z(\varepsilon)$ is equal to the total number of states in the energy shell which is enclosed by the surface $\varepsilon$ $=\varepsilon_{k}$ and $\varepsilon=\varepsilon_{k}-\Delta\left(\varepsilon_{k}\right)$ in $k$-space. Then, the total number of states below an energy $\varepsilon$ in the pure solvent $Z_{0}(\varepsilon)$ differs from that in the dilute alloy $Z_{A}(\varepsilon)$ by an amount which depends on the energy shifts

$$
Z_{A}(\varepsilon)=Z_{0}(\varepsilon)-N_{0}(\varepsilon) \bar{\Delta}(\varepsilon)
$$

where

$$
\vec{\Delta}(\varepsilon)=\frac{2}{(2 \pi)^{3} N_{0}(\varepsilon)} \int_{\varepsilon=\varepsilon_{k}} \frac{d S}{\left|\Delta \varepsilon_{k}\right|} \Delta\left(k, \varepsilon_{k}\right) .
$$

Here, $d S$ is an element of area on the surface $\varepsilon=\varepsilon_{k}$ at the point $k$. The factor of 2 comes from the degree of freedom of a spin. Thus, the density of states is given by

$$
N_{A}(\varepsilon)=N_{0}(\varepsilon)-\frac{d}{d \varepsilon}\left\{N_{0}(\varepsilon) \bar{\Delta}(\varepsilon)\right\}
$$

Since the change in the specific heat of a dilute alloy relative to its value in the pure solvent is proportional to the change in the density of states at the different Fermi-energies, the Fermi energy will be denoted by a variable $\eta$. $\eta_{A}$ and $\eta_{0}$ will be referred to the Fermi energy for the dilute alloys and for the solvent, respectively. Since the electwonic specific heat coefficient, $\gamma$, is proportional to the density of states at Fermi energy, one obtains the following relation,

$$
\begin{aligned}
& \Delta \gamma \propto \Delta N=N_{A}\left(\eta_{A}\right)-N_{0}\left(\eta_{0}\right) \\
& =\left(\eta_{A}-\eta_{0}\right) \frac{d N_{0}\left(\eta_{0}\right)}{d \eta_{0}}+N_{A}\left(\eta_{0}\right)-N_{0}\left(\eta_{0}\right)
\end{aligned}
$$


to the lowest order in the concentration of the solute. From eqs. (2) and (3), one gets

$$
\begin{aligned}
\Delta N & =\left\{\eta_{A}-\eta_{0}-\bar{\Delta}\left(\eta_{0}\right)\right\} \frac{d N_{0}\left(\eta_{0}\right)}{d \eta_{0}} \\
& -N_{0}\left(\eta_{0}\right) \frac{d \bar{\Delta}\left(\eta_{0}\right)}{d \eta_{0}} .
\end{aligned}
$$

Using the same mathematical procedure, one can further derive the change of the total number of states,

$$
Z_{A}\left(\eta_{A}\right)-Z_{0}\left(\eta_{0}\right)=\left\{\eta_{A}-\eta_{0}-\vec{\Delta}\left(\eta_{0}\right)\right\} N_{0}\left(\eta_{0}\right)
$$

Using eqs. (3) and (4), one obtains the equation

$$
\begin{aligned}
\Delta \gamma \propto \Delta N & =\frac{1}{N_{0}\left(\eta_{0}\right)} \frac{d N_{0}\left(\eta_{0}\right)}{d \eta_{0}}\left\{Z_{A}\left(\eta_{A}\right)-Z_{0}\left(\eta_{0}\right)\right\} \\
& -N\left(\eta_{0}\right)-\frac{d \bar{\Delta}\left(\eta_{0}\right)}{d \eta_{0}} .
\end{aligned}
$$

In this relation, $\left\{Z_{A}\left(\eta_{A}\right)-Z_{0}\left(\eta_{0}\right)\right\}$ is the total number of electrons which are added by the solute into the solvent. Therefore, the first term in eq. (5) shows the contribution to the density of states due to the added electrons without changing the shape of the curve. This is the rigid band term.

The second term in eq. (5) is the correction which is caused by the virtual electron scattering in the presence of the solute. If the $\bar{\Delta}(\varepsilon)$ is independent of $\varepsilon$, this term vanishes. A constant $\bar{D}(\varepsilon)$ is a shift of the energy origin on the $N(\varepsilon)$ curve and the specific heat does not depend on the choice of the origin in energy. If $\bar{\Delta}(\varepsilon)$ depends on energy $\varepsilon$, it is difficult to say whether the second term is positive or negative without some specific model to calculate $\bar{\Delta}\left(\eta_{0}\right)$ for each case.

For a ferromagnetic element like $\mathrm{Fe}$, the density of states curve for electron of spin up and of spin down are most likely different. There may be some difference in number of electrons which may go into each of the two bands when the electrons are added by the solute into the solvent. Furthermore, the energy shifts may be different for electrons with spin up and spin down. Thus, we need to extend Brailsford's equation in order to discuss this case. We need to distinguish spin-up and spin-down. We do this by using the symbols $\uparrow$ and $\downarrow$.

The total change of the number of states may be written as

$$
Z_{A} \uparrow\left(\eta_{A}\right)+Z_{A} \downarrow\left(\eta_{A}\right)-Z_{0} \uparrow\left(\eta_{0}\right)-Z_{0} \downarrow\left(\eta_{0}\right)=n \nu c .
$$

where $n$ is the total number of atoms, $\nu$ is the relative valence of the solute with respect to the solvent and $c$ is the concentration of the solute. We write another relation for the relative magnetization $\mu$ of a solute (in Bohr magnetons) with respect to the solvent,

$$
\begin{gathered}
Z_{A} \uparrow\left(\eta_{A}\right)-Z_{0} \uparrow\left(\eta_{0}\right)-Z_{A} \downarrow\left(\eta_{A}\right) \\
+Z_{0} \downarrow\left(\eta_{0}\right)=n \mu c .
\end{gathered}
$$

Eq. (5) can be rewritten twice for spin-up and for spindown. Since $\Delta N=\Delta N \downarrow+\Delta N \uparrow$, we get

$$
\begin{aligned}
\Delta \gamma & \propto \Delta N=\frac{1}{2} \frac{d}{d \eta_{0}}\left[\ln \left\{N_{0} \uparrow\left(\eta_{0}\right) \cdot N_{0} \downarrow\left(\eta_{0}\right)\right\}\right] n \nu c \\
& +\frac{1}{2} \frac{d}{d \eta_{0}}\left[\ln \left\{N_{0} \uparrow\left(\eta_{0}\right) / N_{0} \downarrow\left(\eta_{0}\right)\right\}\right] n \mu c \\
- & {\left[N_{0} \uparrow\left(\eta_{0}\right) \frac{d \bar{d} \uparrow\left(\eta_{0}\right)}{d \eta_{0}}+N_{0} \downarrow\left(\eta_{0}\right) \frac{\overline{d \Delta} \downarrow\left(\eta_{0}\right)}{d \eta_{0}}\right](8) }
\end{aligned}
$$

Here again, the first two terms in eq. (8) are the rigid band terms and the following two terms are the correction terms due to the virtual electron scattering in the presence of the solute. Using eq. (8), we analyze the obtained data which is shown in Fig. 4.

If we assume that the rigid band terms are dominant, one would expect little difference in the $\Delta \gamma$ versus composition relations for $\mathrm{Fe}(\mathrm{Cr}), \mathrm{Fe}(\mathrm{W})$, and $\mathrm{Fe}(\mathrm{Mo})$. The rate of change in $\gamma$ for $\mathrm{Fe}(\mathrm{Mo})$, however, is as much as a half of the value in the other two alloys. The variation in $d \gamma / d c$ for the solutes from the first transition series is rather unsystematic. Corrections for the difference in magnetic moment change with solute are not so essential. For example, the additions of $\mathrm{Mn}$ and $\mathrm{Cr}$ decrease the moment at almost the same rate, yet differ in sign on the effect on $\gamma$. We have not found a scheme which will fit the results of our experiment using only the rigid band terms. Therefore, it seems reasonable to conclude that the energy shift terms are dominant for the dilute solid solution alloys. As some specific models are needed to analyze the present results in detail with the energy shift terms, we do not pursue the analysis further. It seems that the one electron treatment of Brailsford is so comprehensive that it would be premature to consider the effects outside the one electron approximation in treating dilute alloys.

$\mathrm{Fe}(\mathrm{Al})$ and $\mathrm{Fe}(\mathrm{Si})$ systems show some similarity for the low concentration of the solute. Gupta, Cheng and Beck $^{(15)(16)}$ made measurements of these two systems to much higher solute concentrations. These two curves of $\Delta \gamma$ versus solute concentration show that both solutes increase the $\gamma$ value up to a certain concentration of the solutes and begin to decrease the value after passing the peaks at different solute concentration. The $\gamma$ value of $\mathrm{Fe}(\mathrm{Si})$ hits the $\gamma$ value of $\mathrm{Fe}$ at $12 \% \mathrm{Si}$ and for $\mathrm{Fe}(\mathrm{Al})$ at $17 \% \mathrm{Al}$. It has been concluded ${ }^{(15)}$ that this rapid increment in $\gamma$ value is attributed to a magnetic contribution to the low temperature specific heat term which is in a linear relationship with temperature. On the other hand, $\mathrm{Fe}(\mathrm{Si})$ does not show any of the magnetic contributions and the $r$ value stays even below the $\gamma$ value of pure $\mathrm{Fe}$. We have not found any good explanation for the similiar changes in $\gamma$ value for low solute concentrations of $\mathrm{Al}$ and $\mathrm{Si}$.

Finally, as mentioned above, Arrott and Noakes have measured the lattice parameter, spontaneous magnetization, Curie temperature, high temperature susceptibility and electrical resistivity, using the samples taken from the same ingots as used in these specific heat measurements. We have so far found one interesting correla-

(15) K. P. Gupta, C. H. Cheng and P. A. Beck: J. Phys. Chem. Solids, 25 (1964), 1147.

(16) C. H. Cheng, K. P. Gupta and P. A. Beck: J. Phys. Chem. Solids, 25 (1964), 759. 
tion from these measurements. That is, the correlation between the variations of $\gamma$ and Curie temperature with respect to the solute concentration. The four solutes, $\mathrm{Mn}, \mathrm{Ni}, \mathrm{Al}$ and $\mathrm{Si}$, increase the $\gamma$ values and decrease the Curie temperature. On the other hand, five out of the six solutes, Ti, V, Cr, W, Co and Mo, which decrease the $\gamma$ value, increase the Curie temperature. Mo is the only exception for this correlation. Mo decreases both $\gamma$ and Curie temperature ${ }^{(17)}$. We note that $\mathrm{Fe}(\mathrm{Mn})$ and $\mathrm{Fe}(\mathrm{Ni})$ have quite similiar phase diagrams. In addition to the similiar changes in Curie temperature, both show open $\gamma$ loops with the $\alpha-\gamma$ transition temperature decreasing very rapidly with increasing solute concentration. We do not know yet why these two systems are similiar to each other thermodynamically.

The theoretical treatment of the change in $\gamma$ with

(17) S. S. Shinozaki and A. Arrott: J. Appl. Phys., 38 (1967), 1241. alloying is not sufficiently developed to analyze fully the results obtained here. Yet, at least a correlation of the change of the specific heat and the change of the magnetic properties could be pointed out. It is hoped that the detailed data obtained here may stimulate the work toward a more thorough understanding of the meaning of the change of the linear term in specific heat and its relation to the electronic band structure of the transition elements.

\section{Acknowledgments}

The author wishes to express his deepest appreciation to Dr. A. Arrott and Dr. H. Sato for their guidance and discussion given to him during this research and for criticisms of this manuscript. He also expresses his thanks to Dr. J. E. Zimmerman for his help during the construction of the calorimeter. 